\title{
Morphogenesis of the infectious HIV-1 virion
}

\author{
Jun-ichi Sakuragi * \\ Department of Viral Infections, Research Institute for Microbial Diseases, Osaka University, Osaka, Japan
}

\section{Edited by:}

Akio Adachi, The University of

Tokushima Graduate School, Japan

\section{Reviewed by:}

Masako Nomaguchi, The University of Tokushima Graduate School, Japan

Mikako Fujita, Kumamoto University,

Japan

*Correspondence:

Jun-ichi Sakuragi, Department of Viral Infections, Research Institute for

Microbial Diseases, Osaka University,

3-1 Yamadaoka, Suita, Osaka

565-0871, Japan.

e-mail: sakuragi@biken.osaka-u.ac.jp
The virion of HIV-1 is spherical and viral glycoprotein spikes (gp120, gp41) protrude from its envelope. The characteristic cone-shaped core exists within the virion, caging the ribonucleoprotein (RNP) complex, which is comprised of viral RNA, nucleocapsid (NC), and viral enzymes. The HIV-1 virion is budded and released from the infected cell as an immature donut-shaped particle. During or immediately after release, viral protease (PR) is activated and subsequently processes the viral structural protein Gag. Through this maturation process, virions acquire infectivity, but its mechanism and transition of morphology largely remain unclear. Recent technological advances in experimental devices and techniques have made it possible to closely dissect the viral production site on the cell, the exterior - or even the interior - of an individual virion, and many new aspects on virion morphology and maturation. In this manuscript, I review the morphogenesis of HIV-1 virions. I focus on several studies, including some of our recent findings, which examined virion formation and/or maturation processes. The story of novel compound, which inhibits virion maturation, and the importance of maturation research are also discussed.

Keywords: HIV-1, virion, maturation, Gag, RNA, morphology

\section{INTRODUCTION}

The infectious particle of virus (virion) is a very sophisticated device since it needs to be durable enough to protect the packaged viral genome from extracellular nucleases and other obstructions; where it has to be subsequently dissociated appropriately in the target cell to initiate infection.

The virion of the retrovirus, including Human Immunodeficiency Virus type 1 (HIV-1), is spherical with a diameter of about $120 \mathrm{~nm}$ and viral glycoprotein spikes protrude from its envelope. HIV-1 dynamically converts the interior morphology of its particle during particle release, termed maturation. Maturation changes the virion morphology from an immature particle, called the donut-shaped particle, to the mature virion; a particle that is (1) lined with viral matrix proteins (MA), (2) containing a condensed cone-shaped core composed of a viral capsid (CA) which finally encapsidates the (3) RNP complex, comprised of viral RNA, NC, and viral enzymes such as reverse transcriptase (RT) and integrase (IN) (Frankel and Young, 1998). After the maturation, the virus acquires ability to infect adjacent host cells and, thus, the maturation is inevitably essential for particle infectivity. Although much about how the process of virion maturation contributes to achieving infectivity remain unclear, it is a wellaccepted concept that viral infectivity is fully acquired only after complete virion maturation (Bukrinskaya, 2004). Here, I review the morphogenesis of HIV-1 virions, focusing on studies, including our own studies, which have examined the virion formation and/or maturation processes. The mutual relationship between protein processing and virion structure are discussed for a further understanding of how virion morphology plays a key role in the HIV-1 life cycle.

\section{HIV-1 VIRION MATURATION PROCESS}

The construction of the spherical virion of HIV-1 solely driven by the viral gag proteins. The precursor proteins of gag (Pr55) and gag-pol (Pr160) are expressed from an unspliced fulllength viral RNA, which is targeted to the plasma membrane by their N-terminal myristoylation and the adequate accumulation of precursor proteins drive virion assembly beneath the host cell membrane (Ganser-Pornillos et al., 2008). Many features of the virion assembly, budding, and release processes are largely unknown, but many host cell factors are suggested to support and/or actively contribute to these processes (MartinSerrano and Neil, 2011). Several thousands of gag precursors are required to construct the normal virion (Briggs et al., 2004), but budded particle is not fully infectious at its current state. To acquire infectivity, the virion must undergo a maturation process, which is mediated by the precursor protein processing by virally encoded PR (Briggs and Krausslich, 2011). Virion maturation is believed to initiate and complete during or immediately after particle release (Kaplan et al., 1994; Bukrinskaya, 2004), although it still remains unclear what triggers viral PR activation.

The Pr55 polypeptide is composed of four protein domains MA, CA, NC, and p6 - and two spacer peptides, $\mathrm{p} 2$ and $\mathrm{p} 1$. The $\mathrm{N}$-to-C terminal order of the six proteins and peptides are MACA-p2-NC-p1-p6, thus containing five proteolytic cleavage sites to be processed by PR. It was previously demonstrated that the processing rates of the five cleavage sites were not equal (Pettit et al., 1994), where the processing rate of the cleavage site between $\mathrm{p} 2$ and $\mathrm{NC}(\mathrm{p} 2 / \mathrm{NC})$ is the fastest, while $\mathrm{p} 1 / \mathrm{p} 6$ and MA/CA are the second and the third, respectively. The processing rates of the two remaining sites $(\mathrm{CA} / \mathrm{p} 2$ and $\mathrm{NC} / \mathrm{p} 1)$ are much slower than 
those of the three aforementioned sites. As virion maturation is suggested to occur during particle production, it is very difficult to observe the exact maturation process over a time series. Therefore, we recently constructed four sequential HIV-1 Gag mutants in an attempt to generate a sequential series of Gag maturation intermediates and effectively snapshoot the process of virion maturation (Ohishi et al., 2011). We examined the physical states of the RNA viral genome, including dimerization and stability, the ability of endogenous reverse transcription and overall infectivity of particles generated with these mutants. The results are summarized in Figure 1. During the virion maturation process, stabilization of the RNA dimer primes during the primary cleavage (p2-NC) of Pr55 Gag. However, the primary cleavage alone is insufficient and the ensuing cleavages are required for complete the uniform dimerization of viral RNA. Although endogenous virion RT activity was fully acquired at the initial step of maturation, the following processes were necessary for viral DNA production in the infected cell, suggesting that viral RNA/protein maturation plays a critical role for viral infectivity outside the RT process.

\section{MORPHOLOGICAL STUDIES OF VIRION}

It is well-accepted that HIV-1 virion morphology dramatically changes during Pr55 processing. To investigate whether the morphological changes of the virion are correlated with maturation, we examined virion morphological features generated from the four sequential step mutants via electron microscopy (Ohishi et al., 2011). We classified the virion morphologies into four main groups (A, B, C, and D) based on structure of viral core and membrane features (Figure 1). The morphology of all $\triangle \mathrm{PR}$ virions were typical immature particles (Group A) as demonstrated by many previous reports (e.g., Peng et al., 1989). As expected, virions from the Step0 mutant were very similar to those of $\triangle \mathrm{PR}$, containing a thick electron-dense ring devoid of a conical-shaped core, termed a donut-shaped morphology. The morphologies of the WT were classified into two major groups. One was the typical mature particle (Group D), with a conical core surrounded by an envelope. The second was an enveloped particle containing some amorphous structures rather than an obvious core (Group C). Most of the virions from Step1 to 1.1 were immature (Group B), but did not have an apparent

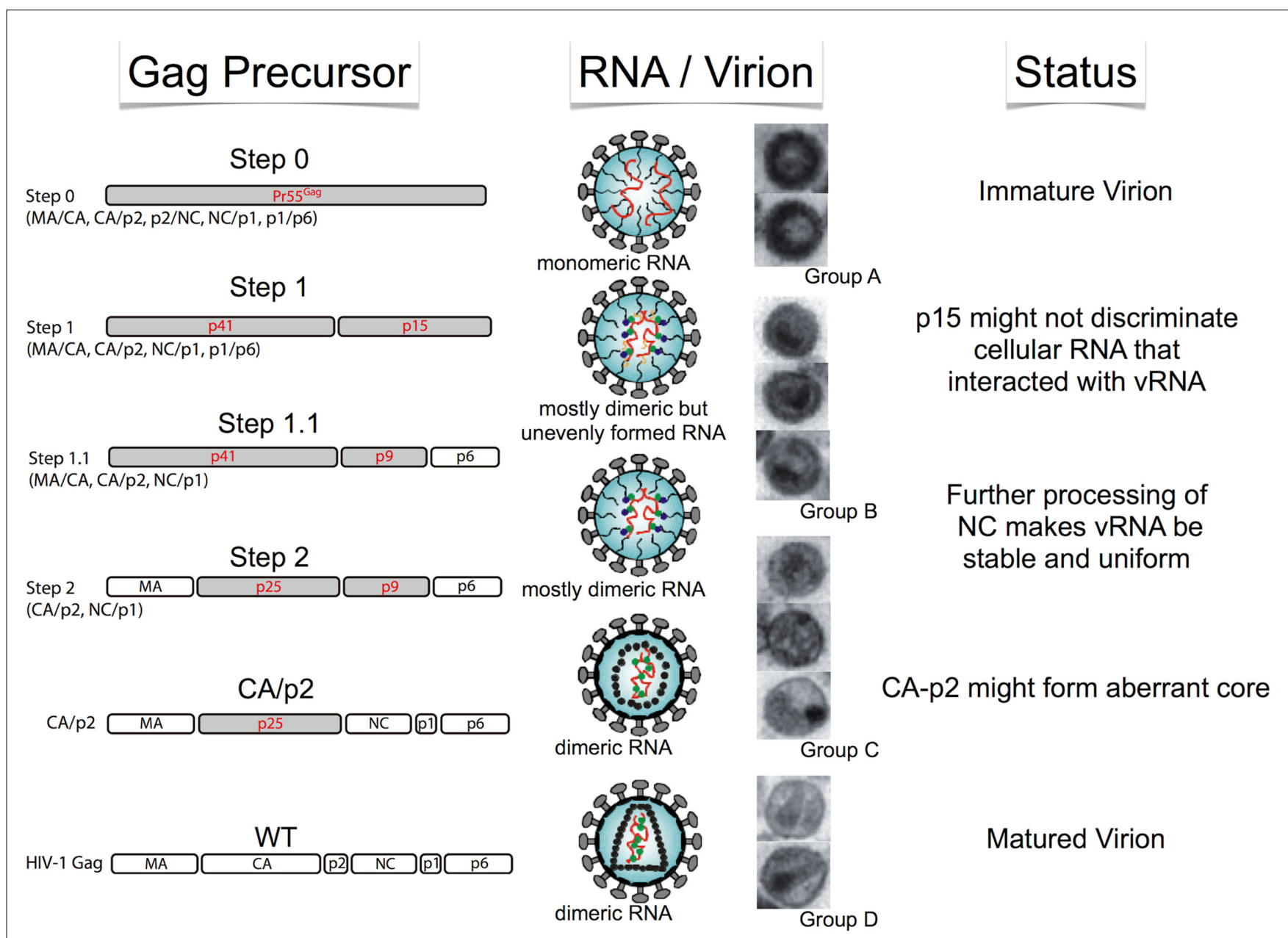

FIGURE 1 | Schematic of morphological and biological transition of the HIV-1 virion during maturation. Stepwise features of Gag cleavage, RNA status, and virion morphology are shown. 
electron-dense ring observed in Group A particles, along with envelopes that were thicker than those of Group C. The virions from Step 2 contained both Group B and C particles. Figure 1 represents the schematic of morphological transition of the HIV1 virion during maturation. As the change from Group A to B was characterized by the disappearance of an electron-dense ring inside the viral membrane, the observed ring is likely composed by the p15 (NC-p1-p6) region within Pr55. After the cleavage at $\mathrm{p} 2-\mathrm{NC}$ position, the ordered array of the p15 region would be lost and p15 would be released from inner viral membrane. This transition was synchronous with initial dimeric RNA stabilization, suggesting the release of genome RNA from a perimembrane localization to a space inside the virion where p15 could trigger dimer maturation. The thin-membraned virions (Group C) became abundant after the third proteolytic processing at the MA/CA region, represented by the Step2 mutant. Viral membranes of Group B may appear to be thick because of the fusion between the Pr55 CA and MA, which lines the inner membrane.

Recent advances in analytical technology, particularly electron microscopy, enabled researchers to study the structure of intact virions and even within the virion itself (Liu et al., 2010), and several morphological studies about HIV-1 virion were reported (Carlson et al., 2010; de Marco et al., 2010; Zhang et al., 2011). Of particular note, a cryoelectron tomography study examined the maturation process of the HIV-1 virion (de Marco et al., 2010). Several stepwise mutants of Gag cleavage were constructed and the changes in virion structure that occurred during maturation were observed. They visualized the arrangement of Gag in three-dimensions (3D) using cryoelectron tomography and sub-tomogram averaging. The condensation of the RNP complex with RNA genome and viral proteins were detected at the initial cleavage event. This inner RNA/protein structure appears to maintain a link with the remaining Gag lattice. Processing on both sides of $\mathrm{CA}-\mathrm{p} 2$ - the main structural module of Gag - is required for disassembly of the immature Gag lattice. The results provided structural correlates of the ordered processing events during HIV-1 maturation and were also consistent in principle with our observations (Ohishi et al., 2011).

Another report studied the budding process of HIV-1 (Carlson et al., 2010). They established experimental systems to study HIV1 budding sites by cryoelectron tomography and observed variable $3 \mathrm{D}$ structures within HIV-1 virions and budding sites. By analyzing these images they showed that the organization of released immature virions is not altered to a large degree from the state of its intracellular assembly. They also identified a novel Gag lattice structure present at viral budding sites. It seemed to be caused by the premature processing of Gag precursors and resulted in the production of dead-end particles. The structures were abundant at viral budding sites in some HIV-1 infected T-cells, suggesting that a crucial control step during virus maturation was lost. They suggested this loss of control might be caused by the host cell environment and a host factor, but the significance of this phenomena was unclear. The actin filaments were often observed around and at the budding sites, and sometimes they appeared stuck to the budding virions.
Although it is not clear whether actin actively contributes to the HIV-1 budding process or not, a recent report denied the effect of HIV-1 replication on the coherency of the actin cytoskeleton (Weichsel et al., 2010). On the other hand, clathrin, a major player in the formation of coated vesicles (Pearse, 1976), was reported to facilitate the morphogenesis of retrovirus particles (Zhang et al., 2011). Clathrin was previously thought to be only passively incorporated into retroviral virions (Hammarstedt and Garoff, 2004). However in this report, abundant amounts of clathrin was shown to be actively and specifically incorporated into retrovirus particles. In several cases, retroviral proteins contain peptide motifs for clathrin recruitment. HIV, SIV, and other murine and simian retroviruses actively packaged clathrin and the prevention of clathrin incorporation caused a variety of defects in the generation of infectious virions, evidenced by viral protein destabilization, inhibition of particle assembly/release, and reduction in virion infectivity. The virion morphology of an SIV mutant lacking the PTAP motif showed grossly abnormal clathrin incorporation. Virusproducing-cells exhibited hemispherical protrusions from their surfaces, but complete spherical particles were hardly observed via scanning electron microscopy. In case of MLV, the virion's ability to saturate the TRIM5alpha (T5a) restriction factor was examined. For efficient elimination of T5a activity, the presence of an unmodified capsid lattice was reported to be required (Pertel et al., 2011). As expected, mutant particles lacking clathrin were approximately 10 -fold less active in the T5a saturation assay to that of the WT. The finding suggested that their cores were not properly formed, or unstable, therefore suggesting that clathrin is important for correct MLV particle morphogenesis. These studies suggested that clathrin might be frequently recruited by retroviruses to assist the correct assembly of infectious virions.

\section{ANTI-VIRAL DRUGS TARGETING FOR VIRION MATURATION}

As the virion maturation process is essential for acquiring viral infectivity, it is a reasonable target for developing anti-viral drugs (Adamson and Freed, 2011). The principal anti-viral target of HIV-1 maturation for many effective drugs was viral PR, (Perno, 2011) however, the emergence of drug-resistant strains in patients undergoing drug treatments appear inevitable. In addition, multiple drug targeting a single protein such as PR often induces the emergence of cross-resistant strains, which have very serious implications for infection dissemination and control (Lefebvre and Schiffer, 2008). Thus, the development of drugs using alternative strategies and targets may be required. An alternative target for a maturation inhibitor is Pr55, which is the substrate of PR (Adamson et al., 2009). The small molecule 3-O-(3', 3'dimethylsuccinyl)-betulinic acid (DSB), also known as bevirimat (BVM), PA-457, or MPC-4326, is the first maturation inhibitor, which potently inhibits HIV-1 replication by blocking a late step of the Gag cleavage pathway, preventing scission at the CA-p2 junction (Li et al., 2003). Although the structural study is still ongoing, it has been demonstrated that BVM is incorporated into assembling virus particles in a Gag-dependent manner (Zhou et al., 2005) and is able to prevent cleavage of CA from p 2 in the context of premature Gag used in virion assembly assembled into virions, but not in free Gag in solution (Li et al., 2003; Sakalian et al., 2006). These data have led to a model where the BVM binding site 


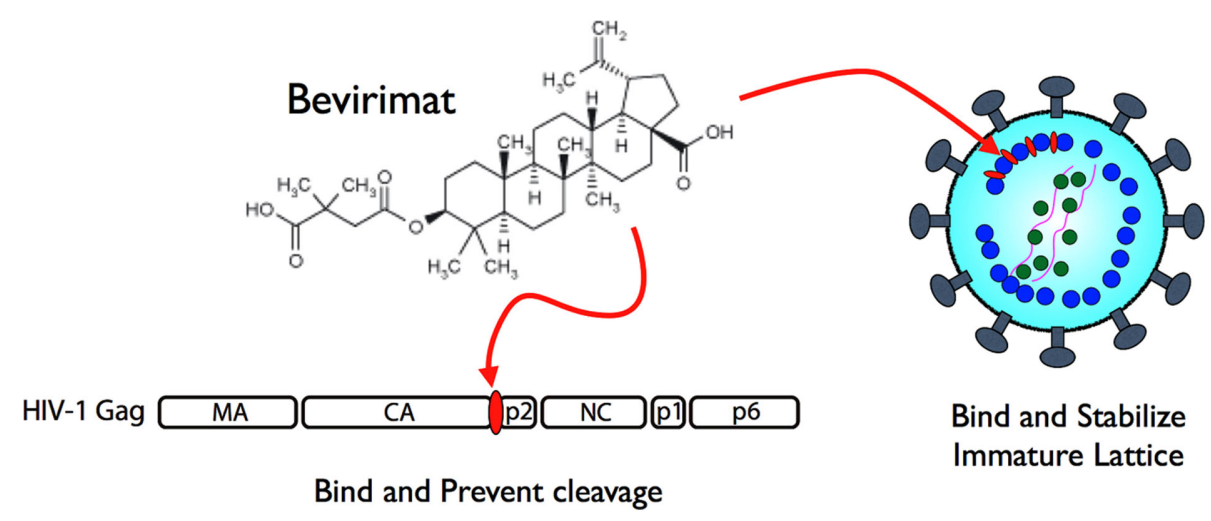

FIGURE 2 | Mechanism of action of bevirimat. It binds to CA/p2 cleavage site of HIV-1 Pr55 to prevent processing by PR (left) and also to stabilize viral core formation in immature status (right).

is present during Gag assembly, at or nearby the CA-p2 cleavage site, and binding of BVM blocks PR from accessing the cleavage site (Figure 2). When virions isolated from BVM-treated cells were observed, the drug disrupted virion production as particles contained an incomplete shell of protein density - with a structure similar to the Gag lattice of an immature HIV-1 particle. Similar to the CA-p2 fusion mutant, BVM binding was suggested to stabilize the immature lattice and prevent capsid maturation, effectively suppressing particle infectivity (Keller et al., 2011). Although BVM was in Phase II clinical trial to determine its use as a treatment against $\mathrm{HIV}-1$, several reports suggested that there was a very low genetic barrier for resistance against BVM (Seclen et al., 2010; Verheyen et al., 2010), eliciting concern for the continuation of its clinical development and subsequently the pharmaceutical company has recently halted BVM development (Wainberg and Albert, 2010). Nonetheless, other pharmaceutical companies still express strong interests in developing a second generation of maturation inhibitor drugs, indicating a compelling demand and promising future for these novel drugs.

\section{CONCLUSION}

The HIV-1 virion structure, as well as the processes involved in their generation, are not fully elucidated. The virion maturation process is one of the most challenging events to be completely understood. It completely changes the morphology and conditions within the virion and primes viral infectivity by properly arranging

\section{REFERENCES}

Adamson, C. S., and Freed, E. O. (2011). Novel approaches to inhibiting HIV-1 replication. Antiviral Res. 85, 119-141.

Adamson, C. S., Salzwedel, K., and Freed, E. O. (2009). Virus maturation as a new HIV-1 therapeutic target. Expert Opin. Ther. Targets 13, 895-908.

Briggs, J. A., and Krausslich, H. G. (2011). The molecular architecture of HIV. J. Mol. Biol. 410, 491-500.

Briggs, J. A., Simon, M. N., Gross, I., Krausslich, H. G., Fuller, S. D., Vogt, V. M., and Johnson, M. C. (2004).
The stoichiometry of Gag protein in HIV-1. Nat. Struct. Mol. Biol. 11, 672-675.

Bukrinskaya, A. G. (2004). HIV-1 assembly and maturation. Arch. Virol. 149, 1067-1082.

Carlson, L. A., de Marco, A., Oberwinkler, H., Habermann, A., Briggs, J. A., Krausslich, H. G., and Grunewald, K. (2010). Cryo electron tomography of native HIV-1 budding sites. PLoS Pathog. 6, el001173. doi:10.1371/journal.ppat.1001173

de Marco, A., Muller, B., Glass, B., Riches, J. D., Krausslich, H. G., and Briggs, J. A. (2010).

the core, which contains the viral RNA and enzymes. Recent advancements of tools and methodologies for experimentation enables scientists to unveil many features of virion morphology and its architecture. Many observations pertaining to virion formation are waiting to be discovered and further structural studies alongside technological improvements would be valuable to resolve these issues. The morphological studies of the virion will not only elucidate the lifecycle of virus replication, but will further the understanding of drug efficacy mechanisms. In fact, cryoelectron tomography studies provided structural correlates of the ordered processing events during HIV-1 maturation and shed light on the mechanism of action of BVM (de Marco et al., 2010). Although current maturation inhibitors seem to be concentrated on the CA-p2 cleavage site (Salzwedel et al., 2007), there remain four additional cleavage sites which may serve as potential drug targets, thus underscoring the importance of studies involving virion morphology and these study cannot be overlooked in the future.

\section{ACKNOWLEDGMENTS}

I appreciate Prof. Tatsuo Shioda and Dr. Seiga Ohmine for critically reading the manuscript. This work was supported by a Grant-inAid for Scientific Research on Priority Areas from the Ministry of Education, Culture, Sports, Science, and Technology (MEXT) of Japan and a grant for HIV/AIDS research from the Ministry of Health, Labor, and Welfare of Japan.

Structural analysis of HIV-1 maturation using cryo-electron tomography. PLoS Pathog. 6, e1001215. doi:10.1371/journal.ppat.1001215

Frankel, A. D., and Young, J. A. (1998). HIV-1: fifteen proteins and an RNA. Annu. Rev. Biochem. 67, 1-25.

Ganser-Pornillos, B. K., Yeager, M., and Sundquist, W. I. (2008). The structural biology of HIV assembly. Curr. Opin. Struct. Biol. 18, 203-217.

Hammarstedt, M., and Garoff, H. (2004). Passive and active inclusion of host proteins in human immunodeficiency virus type $1 \mathrm{gag}$ particles during budding at the plasma membrane. J. Virol. 78, 5686-5697.

Kaplan, A. H., Manchester, M., and Swanstrom, R. (1994). The activity of the protease of human immunodeficiency virus type 1 is initiated at the membrane of infected cells before the release of viral proteins and is required for release to occur with maximum efficiency. J. Virol. 68, 6782-6786.

Keller, P. W., Adamson, C. S., Heymann, J. B., Freed, E. O., and Steven, A. C. (2011). HIV-1 maturation inhibitor bevirimat stabilizes the immature Gag lattice. J. Virol. 85, 1420-1428. 
Lefebvre, E., and Schiffer, C. A. (2008). Resilience to resistance of HIV1 protease inhibitors: profile of darunavir. AIDS Rev. 10, 131-142.

Li, F., Goila-Gaur, R., Salzwedel, K., Kilgore, N. R., Reddick, M., Matallana, C., Castillo, A., Zoumplis, D., Martin, D. E., Orenstein, J. M., Allaway, G. P., Freed, E. O., and Wild, C. T. (2003). PA-457: a potent HIV inhibitor that disrupts core condensation by targeting a late step in Gag processing. Proc. Natl. Acad. Sci. U.S.A. 100, 13555-13560.

Liu, J., Wright, E. R., and Winkler, H. (2010). 3D visualization of HIV virions by cryoelectron tomography. Meth. Enzymol. 483, 267-290.

Martin-Serrano, J., and Neil, S. J. (2011). Host factors involved in retroviral budding and release. Nat. Rev. Microbiol. 9, 519-531.

Ohishi, M., Nakano, T., Sakuragi, S., Shioda, T., Sano, K., and Sakuragi, J. I. (2011). The relationship between HIV-1 genome RNA dimerization, virion maturation and infectivity. Nucleic Acids Res. 39, 3404-3017.

Pearse, B. M. (1976). Clathrin: a unique protein associated with intracellular transfer of membrane by coated vesicles. Proc. Natl. Acad. Sci. U.S.A. 73, 1255-1259.

Peng, C., Ho, B. K., Chang, T. W., and Chang, N. T. (1989). Role of human immunodeficiency virus type 1-specific protease in core protein maturation and viral infectivity. J. Virol. 63, 2550-2556.

Perno, C. F. (2011). The discovery and development of HIV therapy: the new challenges. Ann. Ist. Super. Sanita 47, 41-43.

Pertel, T., Hausmann, S., Morger, D., Zuger, S., Guerra, J., Lascano, J., Reinhard, C., Santoni, F. A., Uchil, P. D., Chatel, L., Bisiaux, A., Albert, M. L., Strambio-De-Castillia, C., Mothes, W., Pizzato, M., Grutter, M. G., and Luban, J. (2011). TRIM5 is an innate immune sensor for the retrovirus capsid lattice. Nature 472 , 361-365.

Pettit, S. C., Moody, M. D., Wehbie, R. S., Kaplan, A. H., Nantermet, P. V., Klein, C. A., and Swanstrom, R. (1994). The p2 domain of human immunodeficiency virus type $1 \mathrm{Gag}$ regulates sequential proteolytic processing and is required to produce fully infectious virions. J. Virol. 68, 8017-8027.

Sakalian, M., McMurtrey, C. P., Deeg, F. J., Maloy, C. W., Li, F., Wild, C. T., and Salzwedel, K. (2006). 3-O-(3', $3^{\prime}$-dimethysuccinyl) betulinic acid inhibits maturation of the human immunodeficiency virus type $1 \mathrm{Gag}$ precursor assembled in vitro. J. Virol. 80, 5716-5722.
Salzwedel, K., Martin, D. E., and Sakalian, M. (2007). Maturation inhibitors: a new therapeutic class targets the virus structure. AIDS Rev. 9, 162-172.

Seclen, E., Gonzalez Mdel, M., Corral, A., de Mendoza, C., Soriano, V., and Poveda, E. (2010). High prevalence of natural polymorphisms in Gag (CA-SP1) associated with reduced response to Bevirimat, an HIV1 maturation inhibitor. AIDS 24, 467-469.

Verheyen, J., Verhofstede, C., Knops, E., Vandekerckhove, L., Fun, A., Brunen, D., Dauwe, K., Wensing, A. M., Pfister, H., Kaiser, R., and Nijhuis, M. (2010). High prevalence of bevirimat resistance mutations in protease inhibitorresistant HIV isolates. AIDS 24 669-673.

Wainberg, M. A., and Albert, J. (2010) Can the further clinical development of bevirimat be justified? AIDS 24, 773-774.

Weichsel, J., Herold, N., Lehmann, M. J., Krausslich, H. G., and Schwarz, U. S. (2010). A quantitative measure for alterations in the actin cytoskeleton investigated with automated highthroughput microscopy. Cytometry A 77, 52-63.

Zhang, F., Zang, T., Wilson, S. J., Johnson, M. C., and Bieniasz, P. D. (2011). Clathrin facilitates the morphogenesis of retrovirus particles. PLoS Pathog. 7, e1002119. doi:10.1371/journal.ppat.1002119

Zhou, J., Huang, L., Hachey, D. L., Chen, C. H., and Aiken, C. (2005). Inhibition of HIV-1 maturation via drug association with the viral Gag protein in immature HIV-1 particles. $J$. Biol. Chem. 280, 42149-42155.

Conflict of Interest Statement: The author declares that the research was conducted in the absence of any commercial or financial relationships that could be construed as a potential conflict of interest.

Received: 15 November 2011; paper pending published: 20 November 2011; accepted: 21 November 2011; published online: 09 December 2011.

Citation: Sakuragi J-i (2011) Morphogenesis of the infectious HIV-1 virion. Front. Microbio. 2:242. doi: 10.3389/fmicb.2011.00242

This article was submitted to Frontiers in Virology, a specialty of Frontiers in Microbiology.

Copyright (c) 2011 Sakuragi. This is an open-access article distributed under the terms of the Creative Commons Attribution Non Commercial License, which permits non-commercial use, distribution, and reproduction in other forums, provided the original authors and source are credited. 